\title{
Negative predictive value of SPECT for the occurrence of MACE in a medium-sized clinic in the Netherlands
}

\author{
M. J. Bom • J. M. B. Manders • R. Uijlings • \\ E. A. Badings - F. M. A. C. Martens
}

Published online: 27 February 2014

(C) The Author(s) 2014. This article is published with open access at Springerlink.com

\begin{abstract}
Background Single-photon emission computed tomography (SPECT) is an important prognostic tool in evaluating coronary artery disease (CAD), with a high negative predictive value (NPV) for the occurrence of major adverse cardiac events (MACE). The prognostic value of SPECT is disputed in women, patients with atrial fibrillation (AF), diabetes, left bundle branch block (LBBB) and renal impairment.

Methods Seven hundred sixty-two patients without prior history of CAD who had SPECT without perfusion deficits were followed for 2 years for MACE. Predictive variables for the occurrence of MACE were reviewed by Cox proportional hazard regression, considering clinical information, restingECG data and SPECT data.

Results The NPV of SPECT for the occurrence of MACE within 2 years was $95.8 \%$. Multivariate Cox regression revealed male gender as the only significant predictor for the occurrence of MACE, besides a positive stress ECG at SPECT and a low LVEF. AF, LBBB, renal impairment and diabetes had no significant effect on the prognosis after normal SPECT.

Conclusion SPECT with normal perfusion images has great NPV in a medium-sized clinic in the Netherlands, even in patients with $\mathrm{LBBB}, \mathrm{AF}$, diabetes and renal impairment. MACE-free survival, however, was negatively influenced by male gender; we therefore propose more caution in men.
\end{abstract}

The questions can be answered after the article has been published in print. You have to log in to: www.cvoi.nl.

M. J. Bom $(\bowtie) \cdot$ R. Uijlings · E. A. Badings · F. M. A. C. Martens Department of Cardiology, Deventer Hospital, N. Bolkesteinlaan 75, 7416 SE Deventer, the Netherlands

e-mail: bommichiel@gmail.com

J. M. B. Manders

Department of Nuclear Medicine, Deventer Hospital, N.

Bolkesteinlaan 75, 7416 SE Deventer, the Netherlands
Keywords Single photon emission computed tomography · SPECT · Prognosis · Major adverse cardiac events · MACE · CAD

\section{Introduction}

Ischaemic heart disease is the second cause of death in the Netherlands, and has a high morbidity and mortality rate [1, 2]. The main cause of myocardial ischaemia is coronary artery disease (CAD) [3]. Single-photon emission computed tomography (SPECT) is an important imaging modality in the analysis of functional ischaemia in patients with suspected $C A D$ [3]. SPECT is reported to have great prognostic value for the occurrence of cardiac events [4-9], with an annual myocardial infarction (MI) rate after negative SPECT of $0.8 \%$ [7]. An important limitation in the use of SPECT is the reduced sensitivity of balanced ischaemia in three-vessel CAD [10].

There have been doubts about the prognostic value of SPECT in certain subgroups. Studies have shown that women [7], patients with renal impairment $(\mathrm{eGFR}<60)$ [11-13] and patients with atrial fibrillation (AF) $[14,15]$ have a higher event rate after normal SPECT. Furthermore, the prognostic use of SPECT in diabetic patients and patients with left bundle branch block (LBBB) has also been disputed.

Recent studies have shown similar event rates after normal SPECT in diabetics compared with non-diabetics [16], although balanced triple-vessel disease is more common in diabetics [17] and thus may cause underestimation of functional ischaemia on SPECT.

Patients with LBBB have intrinsically abnormal conduction and are reported to have a related pattern of perfusion abnormalities. A recent study, however, has shown that prognosis of a normal SPECT was not altered by LBBB [18]. 
Population definition and pre-test likelihood are of great influence on reported prognosis and thus it is crucial to have representable data about our specific population.

\begin{abstract}
Aim
The aim of this study was therefore to evaluate the negative predictive value (NPV) of combined exercise and pharmacological stress SPECT with attenuation correction (AC) in patients without a prior history of CAD in a Dutch hospital, and the influence of above-described subpopulations on the NPV.
\end{abstract}

\section{Methods}

Population

We identified 1491 patients who underwent SPECT between 28 April 2009 and 28 February 2011 in Deventer Hospital. Patients with a prior history of CAD were excluded, as defined by previous MI, revascularisation and/or significant stenosis on coronary angiography, as documented in the medical records. Furthermore, patients with ischaemia and with signs of prior infarction on SPECT were excluded. The remaining total number of studied patients was 762 (Fig. 1).

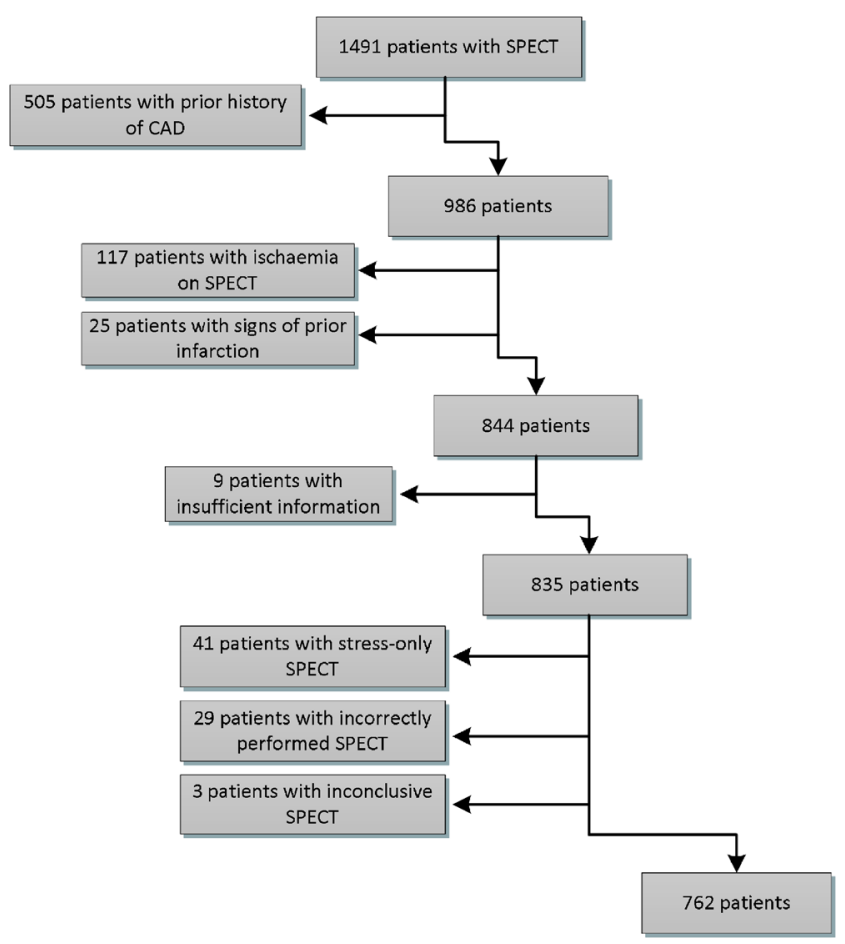

Fig. 1 Inclusion flowchart
Data acquisition

Data were collected from the Electronic Medical Record (EMR) by reviewing letters and reports of the treating cardiologist and other clinicians, reports and letters of clinicians in other hospitals, laboratory results, reports of SPECT by the nuclear medicine physician and the images of SPECT.

Patient preparation and stress protocol

Patient preparation was done according to the American Society of Nuclear Cardiology protocol [19].

Adenosine $(0.14 \mathrm{mg} / \mathrm{kg} / \mathrm{min})$ was infused intravenously for $6 \mathrm{~min}$, and Tc-99m Tetrofosmine was injected after at least $3 \mathrm{~min}$. Adenosine stress was combined with low level treadmill exercise, 20-70 W maximum. Contraindications for exercise were LBBB, paced rhythm and inability to exercise.

Dobutamine stress was performed in patients with severe chronic obstructive pulmonary disease. Dobutamine was administered intravenously, starting at a dose of $10 \mu \mathrm{g} / \mathrm{kg} / \mathrm{min}$. The dose was increased $10 \mu \mathrm{g} / \mathrm{kg} / \mathrm{min}$ every $3 \mathrm{~min}$ until a heart rate of $85 \%$ of the age-predicted maximum heart rate (220-age) was reached, with a maximum of $40 \mu \mathrm{g} / \mathrm{kg} / \mathrm{min}$. A maximum of two additional intravenous injections of atropine $0.5 \mathrm{mg}$ were given.

\section{SPECT acquisition protocol}

Data acquisition was performed using a dual head General Electric Infinia Hawkeye gamma camera (2006) with low energy high resolution collimators. Sixty projections over an angle of $180^{\circ}$ were acquired. Acquisition times for rest and stress were $20 \mathrm{~s}$ and $18 \mathrm{~s}$, respectively, per projection. The energy acceptance window was set at 15-20\%, $140 \mathrm{keV}$ centre. An additional eight frame gated dataset was acquired with an acceptance window of $100 \%$. CT-based AC was used with $140 \mathrm{kV}$ tube voltage and $2.5 \mathrm{mAs}$ tube current. Quantitative gated SPECT software (Cedars-Sinai) was used to automatically calculate end-diastolic volume, end-systolic volume and left ventricular ejection fraction (LVEF). All non-AC images were reconstructed by filtered backward projection, using a 2D Butterworth filter with an order of $8,0.3$ cycles/ pixel. AC and non-AC images were reconstructed by OSEM iterative reconstruction with 2 iterations and 10 subsets.

Patients underwent a 1-day rest-stress acquisition protocol. Rest acquisition was performed 15-30 min after intravenous injection of $250 \mathrm{MBq} \mathrm{Tc}-99 \mathrm{~m}$-tetrofosmine, stress acquisition was performed 15-30 min after intravenous injection of $750 \mathrm{MBq}$ Tc-99m-tetrofosmine. A 2-day protocol was performed in 11 patients because of inadequate patient preparation and in 1 patient because of the low quality of initial stress images. 
Image interpretation

The visual interpretation of perfusion images was based on short-axis and vertical and horizontal long-axis tomograms divided into 20 segments using Myometrix (GE) software. The nuclear medicine physician reviewed all segments, in both $\mathrm{AC}$ and non-AC images, to review the presence of ischaemia.

\section{Follow-up}

The follow-up time was 2 years from the time of inclusion. Patients were followed for major adverse cardiac events (MACE) and all-cause mortality. MACE was defined as fatal and non-fatal MI and revascularisation, consisting of percutaneous coronary intervention (PCI) and coronary artery bypass graft (CABG). MI was defined by typical symptoms and raised troponin-T levels. In 1 case troponin-T was not documented and MI was defined by reviewing the cardiologist letter and the CABG operation report from a tertiary clinic.

\section{Statistical analysis}

Statistical analysis was performed with SPSS software (version 20.0.0, IBM). A $p$-value $<0.05$ was considered statistically significant.

To evaluate the prognosis of patients over time in the different subgroups, univariate and subsequent multivariate Cox proportional hazard regression were performed. Variables included in the univariate analysis consisted of clinical data, resting-ECG data and SPECT data. Selection of variables for entry in the multivariable Cox proportional hazard regression was based on univariate analysis (with a threshold of $p<0.15$ ) and clinical judgment. The multivariate Cox regression was done stepwise according to the backwards approach, with $p<0.10$ as threshold for removal of variables.

The rule of thumb in multivariate Cox regression models is that the events per variable (EPV) must be at least 10 to produce a reliable model [20, 21]. However, a recent study demonstrates that an EPV of 5-10 gives similar reliability to an $\mathrm{EPV} \geq 10$ [22]. Therefore in our study with 32 events, a prediction model may contain 3-6 variables.

The assumption of proportional hazard was tested by obtaining and reviewing the log-minus-log plots, and deemed appropriate in all categories.

To test the linearity assumption of the continuous variable age, martingale residuals were generated from the Cox regression and plotted against age. Assumption of linearity seemed appropriate.

\section{Results}

Study population

The mean age in the 762 included patients was 63.2 years. Of the patients $58.1 \%$ were female and $116(15.2 \%)$ presented with typical chest pain. The average number of risk factors was 2.0. A total overview of clinical data, ECG data and SPECT data is shown in Table 1.

\section{Outcome}

Of the 762 studied patients, 32 (4.2\%) had MACE within follow-up: 1 patient $(0.1 \%)$ died of $\mathrm{MI}, 8$ (1.0 \%) patients had non-fatal MI, in 12 (1.6\%) patients $\mathrm{CABG}$ was performed and 21 (2.8\%) received PCI. The all-cause mortality in our study population was $15(2.0 \%)$. The NPV of SPECT for the occurrence of MACE within 2 years was $95.8 \%$ and the NPV for MI only was $98.8 \%$. An overview of MI, MACE and the NPV of SPECT in the various subgroups is given in Table 2.

Prediction model for the occurrence of MACE

Univariate and subsequent stepwise multivariate Cox proportional hazard regression was performed and the results are presented in Table 3 . The variables with a $p$ value $<0.15$ on univariate analysis and the clinically important variables AF, DM, renal impairment and LBBB were included in the multivariate Cox regression. The only significant independent predictors of MACE were male gender, positive stress ECG on SPECT and low LVEF. AF and hypertension tended to be independent predictors, but were not significant.

\section{Discussion}

Outcome

Our results show that the prognosis of combined stress SPECT with AC without perfusion deficits is very good in patients without a prior history of CAD, with an NPV for the occurrence of MACE within 2 years of $95.8 \%$ and an NPV for MI of $98.8 \%$.

Statistical analysis showed that besides positive stress ECG at SPECT and low LVEF, male gender was the only significant independent predictor for the occurrence of MACE. Thus, the prognosis of a negative SPECT result is not altered by $\mathrm{AF}$, diabetes, $\mathrm{LBBB}$ and renal impairment. 
Table 1 Patient characteristics

\begin{tabular}{ll}
\hline$n=762$ & Number (\%) \\
\hline Clinical data & \\
Age & $63.2 \pm 11.9$ \\
Women & $443(58.1 \%)$ \\
Clinical symptoms & \\
- Typical chest pain & $116(15.2 \%)$ \\
- Atypical chest pain & $444(58.3 \%)$ \\
- No chest pain & $202(26.5 \%)$ \\
Risk factors & \\
- Diabetes & $155(20.3 \%)$ \\
- Hypertension & $389(51.0 \%)$ \\
- Family history $(n=750)^{\mathrm{a}}$ & $315(42.0 \%)$ \\
- Smoking (present and former) & $342(44.9 \%)$ \\
- High cholesterol & $324(42.5 \%)$ \\
Total risk factors $(n=750)^{\mathrm{a}}$ & $2.0 \pm 1.2$ \\
Renal function $(n=739)^{\mathrm{e}}$ & \\
- eGFR $\geq 60$ & $19(2.5 \%)$ \\
- eGFR 30-60 &
\end{tabular}

SPECT data

Type of stress

- Adenosine + exercise

- Adenosine only

- Dobutamine only

- Dobutamine + atropine

- Exercise + atropine

SPECT with AC

Positive exercise ECG at SPECT $(n=760)^{\mathrm{c}}$

Triple vessel disease not excluded ${ }^{\mathrm{e}}$

Summed difference score $($ SDS $)>2$

Functional data

- LVEF $<45(n=758)^{\mathrm{d}}$

$-\mathrm{EDV}>120$

$611(80.2 \%)$
$125(16.4 \%)$
$14(1.8 \%)$
$10(1.3 \%)$
$2(0.3 \%)$
$753(98.8 \%)$
$150(19.7 \%)$
$39(5.1 \%)$
$127(16.5 \%)$
$41(5.4 \%)$
$113(14.8 \%)$

Table 1 (continued)

\begin{tabular}{lc}
$n=762$ & Number $(\%)$ \\
\hline- ESV $>60$ & $89(11.7 \%)$
\end{tabular}

eGFR estimated glomerular filtration rate, Other SVT AV nodal re-entry tachycardia/atrial flutter, ICD implantable cardioverter defibrillator, $A C$ attenuation correction, $L V E F$ left ventricular ejection fraction, $E D V$ enddiastolic volume, ESV end-systolic volume, Triple vessel disease not excluded due to ventricular wall motion abnormalities or abnormal functional data

${ }^{\text {a }}$ In 12 cases family history was not obtained

${ }^{\mathrm{b}}$ eGFR was not obtained in 23 patients

${ }^{\mathrm{c}}$ In two patients stress ECG was not obtained due to technical difficulties

${ }^{\mathrm{d}}$ In four cases LVEF was unreliable, due to AF or GI activity

${ }^{\mathrm{e}}$ Triple vessel disease could not be excluded due to wall motion abnormalities

Overall population

The prognosis after normal SPECT in patients without prior history of CAD has not been previously researched in terms of MACE-free survival. Our study therefore provides key information for the use of SPECT in a medium-sized clinic in the Netherlands. Only two studies are published evaluating the prognosis of a normal SPECT in patients without prior history of CAD in terms of MI rate. These studies report an annual MI rate of $0.6 \%$ following a normal SPECT result $[6,23]$, which is in accordance with the MI rate of $1.2 \%$ within 2 years found in our study.

\section{Subgroups}

The greater prognostic value of SPECT in women compared with men, as seen in our study, has not previously been reported. Prior studies even show that women have a slightly higher event rate than men after normal SPECT [7]. These studies are predominantly performed in large clinics outside

Table 2 Outcome and negative predictive value

\begin{tabular}{lcrl}
\hline & MI $(\%)$ & MACE $(\%)$ & NPV for MACE \\
\hline Overall & $9(1.2 \%)$ & $32(4.2 \%)$ & $95.8 \%$ \\
Women & $1(0.2 \%)$ & $8(1.8 \%)$ & $98.2 \%$ \\
AF & $2(1.9 \%)$ & $2(1.8 \%)$ & $98.2 \%$ \\
DM & $2(1.3 \%)$ & $9(5.8 \%)$ & $94.2 \%$ \\
LBBB & $1(1.9 \%)$ & $4(7.7 \%)$ & $92.3 \%$ \\
Renal impairment & $2(2.7 \%)$ & $4(5.3 \%)$ & $94.7 \%$ \\
Severe renal impairment & $1(5.9 \%)$ & $2(11.8 \%)$ & $88.2 \%$
\end{tabular}

$A F$ atrial fibrillation (prior history/on ECG at presentation), $D M$ diabetes mellitus, $L B B B$ left bundle branch block, renal impairment eGFR $<60$, severe renal impairment $\mathrm{eGFR}<30$ 
Table 3 Univariate and subsequent multivariate Cox regression of clinical data, ECG findings and SPECT data on MACE

\begin{tabular}{|c|c|c|c|}
\hline Variable & HR & $95 \% \mathrm{CI}$ & $P$-value \\
\hline \multicolumn{4}{|l|}{ Univariate analysis } \\
\hline Male gender & 4.266 & $1.916-9.496$ & $<0.001 *$ \\
\hline Age & 1.021 & $0.990-1.052$ & 0.189 \\
\hline Typical chest pain & 1.885 & $0.847-4.196$ & $0.120 *$ \\
\hline $\mathrm{DM}$ & 1.523 & $0.705-3.292$ & 0.285 \\
\hline Hypertension & 1.856 & $0.895-3.850$ & $0.097 *$ \\
\hline Family history & 1.222 & $0.610-2.447$ & 0.572 \\
\hline Smoking & 1.088 & $0.544-2.179$ & 0.811 \\
\hline High cholesterol & 1.189 & $0.594-2.380$ & 0.625 \\
\hline Renal impairment & 1.272 & $0.446-3.625$ & 0.653 \\
\hline Severe renal impairment & 2.917 & $0.697-12.207$ & $0.143^{*}$ \\
\hline $\mathrm{AF}$ & 0.403 & $0.096-1.687$ & 0.213 \\
\hline ECG: prior MI & 1.330 & $0.182-9.744$ & 0.779 \\
\hline ECG: ischaemia & 0.535 & $0.163-1.755$ & 0.302 \\
\hline ECG: LBBB & 2.021 & $0.709-5.761$ & 0.188 \\
\hline Adenosine only & 0.799 & $0.327-1.955$ & 0.623 \\
\hline Stress ECG at SPECT & 2.257 & $1.082-4.711$ & $0.030^{*}$ \\
\hline LVEF $<45 \%$ & 4.369 & $1.798-10.615$ & $0.001 *$ \\
\hline $\mathrm{ESV}>60$ & 2.165 & $0.937-5.006$ & $0.071^{*}$ \\
\hline $\mathrm{EDV}>120$ & 3.110 & $1.500-6.451$ & $0.002 *$ \\
\hline Triple vessel disease not excluded & 3.638 & $1.401-9.448$ & $0.008^{*}$ \\
\hline \multicolumn{4}{|l|}{ Multivariate analysis } \\
\hline Male gender & 4.710 & $2.054-10.801$ & $<0.001$ \\
\hline Hypertension & 1.960 & $0.936-4.105$ & 0.074 \\
\hline Stress ECG at SPECT & 3.079 & $1.460-6.495$ & 0.003 \\
\hline $\mathrm{LVEF}<45 \%$ & 3.967 & $1.584-9.934$ & 0.003 \\
\hline $\mathrm{AF}$ & 0.303 & $0.071-1.294$ & 0.107 \\
\hline
\end{tabular}

$L V E F$ left ventricular ejection fraction, $E D V$ end-diastolic volume, $E S V$ end-systolic volume, $D M$ diabetes mellitus, $A F$ atrial fibrillation (prior history/on ECG at presentation), $M I$ myocardial infarction, $L B B B$ left bundle branch block, Renal impairment eGFR $<60$, severe renal impairment $\mathrm{eGFR}<30$

*: variables with $p<0.15$ on univariate analysis

the Netherlands. Our results may be more representative for clinics in the Netherlands. Research in the Leiden University Medical Centre showed a worse prognosis of MACE in 5.9\% of patients [24]. This study, however, was performed in a higher risk population and with a broader definition of MACE. The worse prognosis in men is an important finding for the daily practice in our hospital and in comparable clinics. Clinicians should be more careful in interpreting normal SPECT in men.

Unlike our results, prior studies show a higher event rate in AF patients after normal SPECT than in non-AF patients [15]. These studies defined AF only by findings on the baseline resting ECG. Both paroxysmal and persistent $\mathrm{AF}$ are known to have increased risk of developing MACE [25, 26].
Therefore, we also included patients with a prior history of AF to analyse the NPV of SPECT in AF patients. Our study is the first to show that the prognosis of normal SPECT in AF patients (on baseline resting ECG and prior history) is comparable with that of non-AF patients.

Patients with renal impairment $(\mathrm{eGFR}<60)$ are reported to have a worse prognosis after normal SPECT than patients with normal renal function [11-13]. Our study, however, did not show any difference in MACE-free survival between these patients. Although patients with severe renal impairment $(\mathrm{eGFR}<30)$ seemed to have a worse prognosis, with MACE in $11.8 \%$ of patients, no statistically significant difference was proven.

The similar prognosis of diabetics and non-diabetics after normal SPECT is in accordance with prior studies [16]. However, the event rate after normal SPECT, although initially similar, is reported to increase after 2 years of follow-up and thus a shorter warranty period of SPECT may apply in diabetic patients [16]. One must be cautious about using our data to predict the long-term prognosis of a normal SPECT in diabetic patients.

Patients with LBBB are known to have an abnormal conduction pattern, associated with abnormal perfusion images. When this is taken into account, patients with LBBB are reported to have a similar prognosis after normal SPECT to patients without LBBB [18]. Although the prognosis seemed worse, with MACE in $7.7 \%$ of LBBB patients, compared with $2.6 \%$ in non-LBBB patients, no difference in MACEfree survival was found. However, the small cohort of 52 LBBB patients limits the extrapolation of results and therefore we advise clinicians to be cautious when reviewing normal SPECT in LBBB patients.

\section{Limitations}

Recently, software has been developed to automatically quantify perfusion in SPECT. The automated quantitative scores are reported to have greater prognostic value [27] and reproducibility than visual assessment [28]. In our study, perfusion images of SPECT were visually assessed, because local experience with the visual approach was greater than with automated quantitative software. Although SPECT is known to have little intra- and interobserver variability in experienced observers [3, 29, 30], the reliance upon the expertise of the observer limits the extrapolation of our results to other centres.

The definition of MI was also subject to inter-observer variability, because the cardiologist's interpretation and clinical judgement played an important role in determining whether the patient presented with classical signs of MI.

Our population of 762 patients was sufficient to investigate the NPV of a negative SPECT in the overall population, 
despite the limited number of events. However, the number of patients in the various subgroups was often small. To prove statistically significant differences in MACE-free survival in subgroups larger study populations are needed, especially in patients with LBBB and patients with severe renal impairment. The limited number of events also compromised the reliability of the prediction model generated by the Cox proportional hazard regression.

\section{Conclusion}

Our study shows that the negative predictive value of combined stress SPECT with AC on the occurrence of MACE within 2 years in patients without prior CAD in a mediumsized clinic in the Netherlands is very good, even in patients with $\mathrm{AF}$, diabetes and moderate renal impairment. However, caution is advised when reviewing SPECT in men, patients with LBBB and patients with severe renal impairment. Statistical analysis showed significantly worse MACE-free survival in men. MACE-free survival also seemed worse in patients with LBBB and patients with severe renal impairment, but this was not significant. Further prospective research is needed in these patients.

Funding This work was not supported with any grants and did not receive funding of any kind.

\section{Conflict of interest None declared.}

Open Access This article is distributed under the terms of the Creative Commons Attribution License which permits any use, distribution, and reproduction in any medium, provided the original author(s) and the source are credited.

\section{References}

1. Centraal Bureau voor de Statistiek. Doodsoorzaken; maand en jaar van overlijden. 2013. Available at: http://statline.cbs.nl/StatWeb/ publication $/$ ? VW $=\mathrm{T} \& \mathrm{DM}=\mathrm{SLNL} \& \mathrm{PA}=71594$ ned \&LA $=\mathrm{NL}$. Accessed 15-01, 2013.

2. Murray CJ, Lopez AD. Evidence based health policy-lessons from the Global Burden of Disease Study. Science. 1996;274:740-3.

3. Fox K, Alonso Garcia MA, Ardissino D, et al. Guidelines on the management of stable angina pectoris: executive summary. The Task Force on the Management of Stable Angina Pectoris of the European Society of Cardiology. Eur Heart J. 2006;27:1341-81.

4. Mowatt G, Brazzelli M, Gemmellb H, et al. Systematic review of the prognostic effectiveness of SPECT myocardial perfusion scintigraphy in patients with suspected or known coronary artery disease and following myocardial infarction. Nucl Med Commun. 2005;26:21729.

5. Schinkel AFL, Elhendy A, Van Domburg RT, et al. Incremental value of exercise technetium- $99 \mathrm{~m}$ tetrofosmin myocardial perfusion single- photon emission computed tomography for the prediction of cardiac events. Am J Cardiol. 2003;91:408-11.

6. Shaw LJ, Hendel R, Borges-Neto S, et al. Prognostic value of normal exercise and adenosine 99mTc-tetrofosmin SPECT imaging: results from the multicenter registry of 4,728 patients. Nucl Med. 2003;44: 134-9.

7. Shaw LJ, Iskandrian AE. Prognostic value of gated myocardial perfusion SPECT. J Nucl Cardiol. 2004;11:171-85.

8. Jain D, Lessig H, Patel R, et al. Influence of $99 \mathrm{mTc}$-tetrofosmin SPECT myocardial perfusion imaging on the prediction of future adverse cardiac events. J Nucl Cardiol. 2009;16:540-8.

9. Boiten HJ, van der Sijde NJ, Ruitinga PR, et al. Long-term prognostic value of exercise technetium-99m tetrofosmin myocardial perfusion single-photon emission computed tomography. J Nucl Cardiol. 2012;19:907-13.

10. Beller GA. Underestimation of coronary artery disease with SPECT perfusion imaging. J Nucl Cardiol. 2008;15:151-3.

11. Hakeem A, Bhatti S, Dillie KS, et al. Predictive value of myocardial perfusion single-photon emission computed tomography and the impact of renal function on cardiac death. Circulation. 2008;118: 2540-9.

12. Al-Mallah $\mathrm{MH}$, Hachamovitch R, Dorbala S, et al. Incremental prognostic value of myocardial perfusion imaging in patients referred to stress single-photon emission computed tomography with renal dysfunction. Circ Cardiovase Imaging. 2009;2:429-36.

13. Okuyama C, Nakajimab K, Hattac T, et al. Incremental prognostic value of myocardial perfusion single photon emission computed tomography for patients with diabetes and chronic kidney disease. Nucl Med Commun. 2011;32:913-9.

14. Askew JW, Miller TD, Hodge DO, et al. The value of myocardial perfusion single-photon emission computed tomography in screening asymptomatic patients with atrial fibrillation for coronary artery disease. J Am Coll Cardiol. 2007;50:1080-5.

15. Abidov A, Hachamovitch R, Rozanski A, et al. Prognostic implications of atrial fibrillation in patients undergoing myocardial perfusion single-photon emission computed tomography. J Am Coll Cardiol. 2004;44:1062-70.

16. Giri S, Shaw LJ, Murthy D, et al. Impact of diabetes on the risk stratification using stress single-photon emission computed tomography myocardial perfusion imaging in patients with symptoms suggestive of coronary artery disease. Circulation. 2002;105:32-40.

17. Fallow GD, Singh J. The prevalence, type and severity of cardiovascular disease in diabetic and non-diabetic patients: a matched-paired retrospective analysis using coronary angiography as the diagnostic tool. Mol Cell Biochem. 2004;261:263-9.

18. Ten Cate TJF, Kelder JC, Plokker HWM, et al. Myocardial perfusion SPECT identifies patients with left bundle branch block patterns at high risk for future coronary events. J Nucl Cardiol. 2010;17:216-24.

19. Henzlova MJ, Cerqueira MD, Mahmarian JJ, et al. Stress protocols and tracers. J Nucl Cardiol. 2006;13:e80-90.

20. Peduzzi P, Concato J, Kemper E, et al. A simulation study of the number of events per variable in logistic regression analysis. J Clin Epidemiol. 1996;49:1373-9.

21. Concato J, Peduzzi P, Holfold TR, et al. Importance of events per independent variable in proportional hazards analysis. I. Background, goals, and general strategy. J Clin Epidemiol. 1995;48:1495-501.

22. Vittinghoff $\mathrm{E}, \mathrm{McCulloch} \mathrm{CE}$. Relaxing the rule of ten events per variable in logistic and Cox regression. Am J Epidemiol. 2007;165: $710-8$.

23. Hachamovitch R, Hayes S, Friedman JD, et al. Determinants of risk and its temporal variation in patients with normal stress myocardial perfusion scans: what is the warranty period of a normal scan? J Am Coll Cardiol. 2003;41:1329-40.

24. America YG, Bax JJ, Boersma E, et al. The additive prognostic value of perfusion and functional data assessed by quantitative gated SPECT in women. J Nucl Cardiol. 2009;16:10-9. 
25. Weijs B, de Vos CB, Tieleman RG, et al. The occurrence of cardiovascular disease during 5-year follow-up in patients with idiopathic atrial fibrillation. Europace. 2013;15:18-23.

26. Osranek M, Bursi F, Bailey KR, et al. Left atrial volume predicts cardiovascular events in patients originally diagnosed with lone atrial fibrillation: three-decade follow-up. Eur Heart J. 2005;26:2556-61.

27. Leslie WD, Tully SA, Yogendran MS, et al. Prognostic value of automated quantification of $99 \mathrm{mTc}$-sestamibi myocardial perfusion imaging. J Nucl Med. 2005;46:204-11.
28. Xu Y, Hayes S, Ali I, et al. Automatic and visual reproducibility of perfusion and function measures for myocardial perfusion SPECT. J Nucl Cardiol. 2010;17:1050-7.

29. Garcia EV. SPECT attenuation correction: an essential tool to realize nuclear cardiology's manifest destiny. J Nucl Cardiol. 2007;14:1624.

30. Golub RJ, Ahlberg AW, McClellan JR, et al. Should SPET attenuation correction be more widely employed in routine clinical practice? For Eur J Nucl Med Mol Imaging. 2002;29: 409-12.

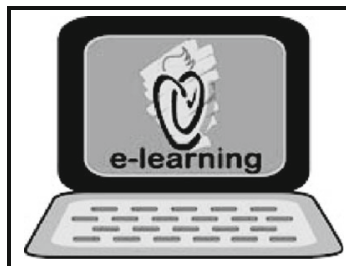

\section{CVOI E-learning formula!}

This is the CVOI e-learning article. The author has prepared 10 questions which are available through the website of the Cardiovascular Educational Institute (CVOI). Please follow the instructions below.

After finishing the questions you will be asked to fill in your name, hospital and e-mail address; then press the button 'verzenden'.

When 6 out of the 10 questions are answered correctly, you acquire 1 accreditation point granted by the Quality Committee of the Netherlands Society of Cardiology (NVVC). The acquired point will be credited to your personal file in the GAIA system. You will also receive an e-mail with all the correct answers.

Over a period of one year 10 e-learning articles will appear in 10 subsequent NHJ editions. In each edition the e-learning article will be recognisable by a special icon. On an annual basis you can collect 10 accreditation points. The accreditation points are credited in the GAIA system by the CVOI.

If you need additional information, please contact the CVOI by e-mail: cvoi@cvoi.org or by phone: 030-2345001.

E.E. van der Wall

Chief editor NHJ
K.B. Schick

Coordinator CVOI 